\title{
New cLFV search experiments using the $\mu-e$ conversion process
}

\section{Satoshi Mihara'}

High Energy Accelerator Research Organization (KEK)

1-1 Oho Tsukuba, Ibaraki 305-0081, Japan

E-mail: satoshi.mihara@kek.jp

Charged lepton-flavor violation (cLFV) searches are thought to be sensitive to a new physics beyond the standard model. Recently, the LHC (the high-energy frontier) and MEG (which has performed the highest sensitive measurement of a muon-rare decay) started physics data acquisition and are expected to provide exciting results in the near future. In this presentation, future muon-based cLFV experiments that are complementary to these experiments will be reviewed along with a brief introduction to the physics motivation of cLFV searches.

The Xth Nicola Cabibbo International Conference on Heavy Quarks and Leptons,

Frascati (Rome), Italy

October 11-15, 2010

$1 \quad$ Speaker 


\section{Introduction}

The muon has played an important role in building the standard model (SM) of particle physics. Many experiments searching for exotic muon decays have yielded negative results; this has led to the development of the concept of lepton-flavor conservation. Currently, the search for possible flavor violation of the muon is considered to be a powerful and the most sensitive tool to reveal or restrict a new physics beyond the SM.

\subsection{Muon in particle physics}

The muon was discovered in cosmic rays in 1936 [1]. At that time, it was considered that the muon would decay to an electron and a neutrino; however, this supposition was later found to be inconsistent with experimental observation. Hincks and Pontecorvo performed an experiment to examine another hypothesis, that is, the muon decaying to an electron and a gamma; this examination was eventually recognized as the first charged lepton-flavor violation (cLFV) search experiment [2]. In 1958 Feinberg concluded that a theoretical calculation of the $\mu \rightarrow e \gamma$ branching ratio with a single-flavor neutrino was not consistent with the experimental upper limit achieved at that time, resulting in the introduction of the two-flavor neutrino model [3]. This was confirmed in an experiment in 1958 [4], which led to the conclusion that the muon neutrino is different from the electron neutrino; thus, the concept of lepton-flavor conservation was established in the SM.

\subsection{Muon flavor physics}

Because the lepton-flavor conservation has been established in the SM, any indication of its violation will be stunning evidence of the existence of physics beyond the SM. Though the SM can describe almost all known phenomena of elementary particles, it has been pointed for many years that the SM itself is not a complete model and needs correction so that the model does not contain any unnatural fine tuning. One of the models that incorporate such a correction is the supersymmetric extension of the SM. In this model, generally speaking, the grand unification of matter and forces can be naively realized somewhere around $10^{16} \mathrm{GeV}$. Currently, we know that quarks are mixed and details are well described by the Cabibbo-KobayashiMaskawa matrix, and that neutrinos (neutral leptons) are mixed, as proven by neutrinooscillation experiments. These facts suggest that the charged leptons are also mixed based on the grand-unification theory. Many theoretical calculations actually indicate that the cLFV may be observed in the muon just below the current experimental bounds $[5,6,7]$.

\section{Charged lepton-flavor violation in muon decays}

Among many theoretical predictions on possible cLFV processes, the muon system is thought to be most sensitive to the new physics because of two reasons: a high muon production rate and long muon lifetime. A leading muon cLFV experiment is the MEG experiment at the Paul Scherrer Institute (PSI) in Switzerland, which is searching for $\mu \rightarrow e \gamma$ decays with a sensitivity of $10^{-13}$ [8]. Other attempts are in progress in both Japan and the US. The COMET 
experiment plans to reach a sensitivity to the $\mu-e$ conversion process of $10^{-16}$ using a highintensity pulsed muon beam provided at the Japan Proton Accelerator Research Complex (JPARC) in Japan [9], and the Mu2e experiment is aiming at achieving a similar sensitivity by using a pulsed-muon beam produced at the Fermi National Laboratory (FNAL) accelerators in the US [10]. Both $\mu \rightarrow e \gamma$ and $\mu-e$ conversion are thought to occur based on the same physics origin as depicted in Figure 1. When photon exchange (Figure 1(b)) dominates the $\mu-e$ conversion process, the ratio of branching ratios of the two processes will be of the order of $\alpha$, the fine structure constant, because of the additional coupling of the photon to the quark line in the $\mu-e$ conversion process. If any other particle exchange contributes to the $\mu-e$ conversion process in addition to the photon exchange, the ratio of branching ratios can be well larger than $\alpha$. Thus, measurements of the branching ratio of both the processes will provide us

(a)

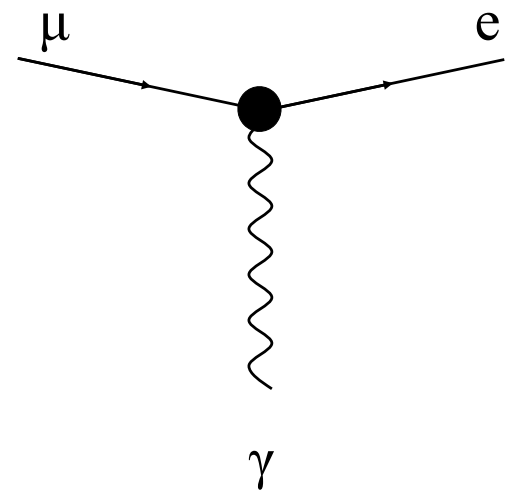

(b)

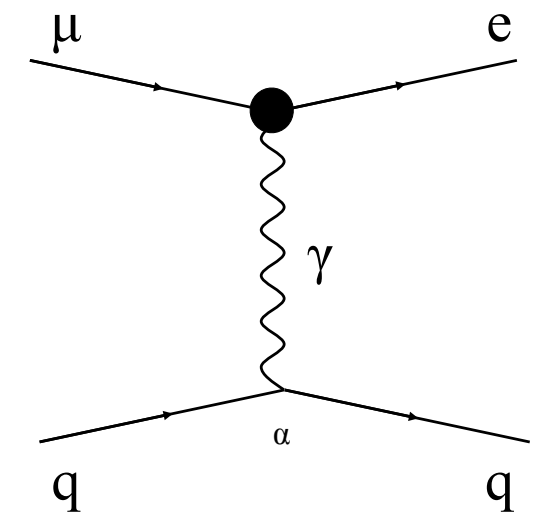

Figure 1: Feynman diagrams of (a) $\mu \rightarrow e \gamma$ and (b) $\mu-e$ conversion. with important information to discriminate the physics contributing to these processes.

\section{Mu-e conversion search experiments}

The current best limit of the $\mu-e$ conversion search was obtained in the SINDRUM II experiment: $7 \times 10^{-13}$ at a $90 \%$ confidence level [11]. The experiment was performed at the PSI, where an intense direct-current muon beam produced at a rate of more than $10^{8} \mu / \mathrm{s}$ is available. Currently, three experiments are planned in the US and Japan; these experiments are competing with each other in an effort to realize an experiment with a sensitivity higher than the SINDRUM II limit.

\subsection{Mu2e at FNAL}

The Mu2e experiment plans to utilize the FNAL accelerator chain after Tevatron shutdown. The protons accelerated up to $8 \mathrm{GeV} / \mathrm{c}$ in the booster are transferred to the debuncher ring through the main injector/recycler. The proton beam time structure is reformed in the debuncher 
ring to achieve a pulse duration shorter than $100 \mathrm{~ns}$. The circumference of the debuncher is 1.7 $\mu \mathrm{s}$, which is approximately twice the muon lifetime in a muonic aluminum atom, and thus suitable to realize a pulse separation for the $\mu-e$ conversion search experiment. The proton beam is then transferred to the accumulator ring for extraction to the experimental area where the pion production target, muon stopping target, and electron spectrometer are located.

Figure 2 shows a schematic view of the setup in the experimental area. The extracted proton beam from the accumulator ring is injected into a target located on the central axis of a solenoid magnet with a gradient magnetic field; this arrangement enables us to collect low momentum pions in the backward direction. These pions decay to muons during transportation through an S-shaped solenoid magnet. Inside the S-shape transport solenoid magnet, a collimator is located, which eliminates muons with high momentum that cannot be stopped in the muon stopping target. The muon stopping target, which is made of aluminum at the initial stage of the experiment and will be exchanged for a heavier material in the future, is located at the end of the transport solenoid. The muons that are stopped in this target form muonic atoms and decay in the orbit or are captured by nuclei followed by neutrino emissions. In either case, the lepton-flavor number is conserved owing to the neutrino emission. However, if the $\mu-e$ conversion occurs, no neutrino is emitted and only a single electron comes out from the target with a typical energy of $105 \mathrm{MeV}$, which is equivalent to the muon mass minus the binding energy of a muon in a muonic aluminum atom. Electrons will be measured by a spectrometer connected to the transport solenoid. This is composed of a solenoid magnet and tracker for momentum analysis, and a crystal calorimeter for energy measurement.

The Mu2e experiment requires a beam power of $20 \mathrm{~kW}$ to reach a single-event sensitivity of $2.5 \times 10^{-17}$. It is also necessary to suppress the number of remaining protons between beam pulses, which would cause inevitable physics background for the experiment, to a value less than $10^{-9}$ compared to the number of protons in a pulse. This ratio is called the extinction factor of the proton beam.

\section{- Tracking and Calorimeter}

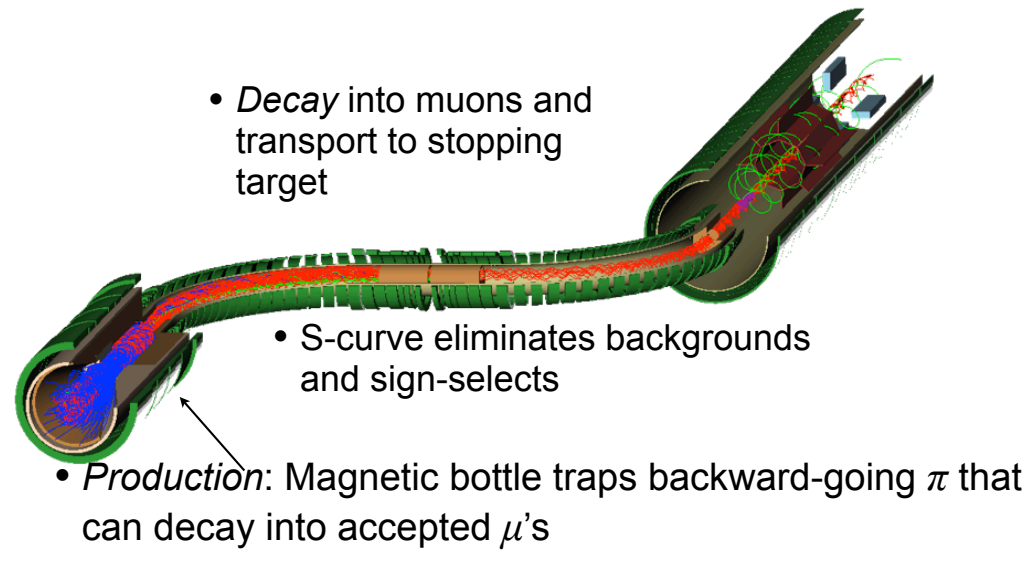

Figure 2: Setup of the Mu2e experiment 


\subsection{COMET at J-PARC}

The COMET experiment searches for the $\mu-e$ conversion with a sensitivity of $10^{-16}$ at a $90 \%$ confidence level upper limit. The experiment uses the J-PARC main ring (MR) accelerator to realize a required beam structure as in the case of the Mu2e experiment. The J-PARC MR employs an RF frequency of $1.7 \mathrm{MHz}$ for beam acceleration with a circumference of $5.3 \mu \mathrm{s}$, providing a beam pulse separation of $0.59 \mu \mathrm{s}$ in its normal operation. For the COMET experiment, which requires a pulse separation as large as the muon lifetime in a muonic aluminum atom $(0.88 \mu \mathrm{s})$, the MR will be operated by filling protons in every other acceleration bucket. This acceleration scheme affords a suitable time structure for a pulse separation (1.2 $\mu \mathrm{s}$, or $1.8 \mu \mathrm{s}$ when every third acceleration bucket is filled). The proton beam accelerated to $8 \mathrm{GeV}$ is extracted with keeping this time structure to the experimental area where particle and nuclear physics experiments are performed.

Figure 3 shows a schematic view of the COMET experimental setup. The pion production target is located within a solenoid magnet with a graded field. Pions produced in the backward direction are mainly transferred and decay to muons during transportation. Muons are transported though a U-shape solenoid magnet for momentum selection with a collimator at its end. A muon stopping target, composed of arranged aluminum disks, is located in a target solenoid followed by another U-shape solenoid magnet for electron momentum selection. The momentum and energy of electrons are measured by a tracker and crystal calorimeter located at the end of the spectrometer solenoid.

The single-event sensitivity of the experiment is $2.6 \times 10^{-17}$, assuming that a beam extinction of less than $10^{-9}$ is successfully realized. Currently, the COMET collaboration is working out the details of the technical design of the experiment and is conducting R\&D work on the primary proton beam, superconducting solenoid magnet, muon stopping target, and electron detectors. The collaboration plans to complete the construction of the experimental setup sometime around 2015. Then, an engineering run will be carried out for one year, after which physics data acquisition will continue.

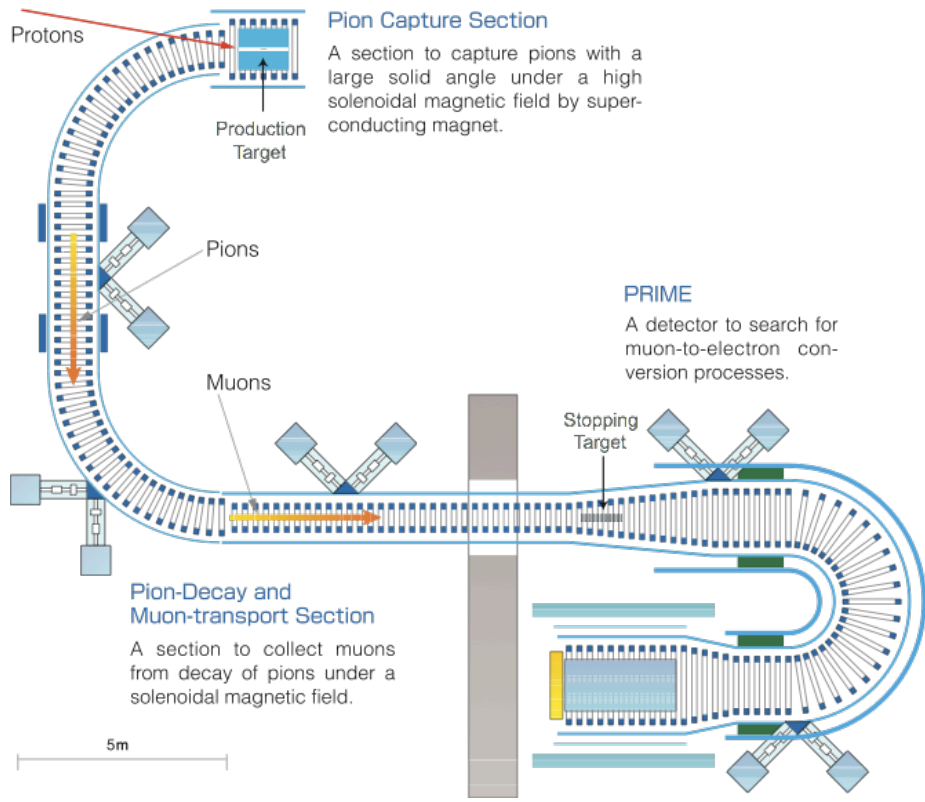

Figure 3: Setup of the COMET experiment. 


\subsection{DeeMe at J-PARC}

Another attempt is being made at J-PARC to search for the $\mu-e$ conversion process. The target sensitivity of the DeeMe experiment [12] is two orders of magnitude larger than that of the Mu2e and COMET experiments. However the DeeMe experiment may have a higher chance of discovery thanks to its lower cost and shorter period of construction provided the branching ratio is sufficiently high. The experiment utilizes the proton beam provided by the JPARC RCS accelerator to the Materials and Life Science Facility (MLF); in MLF, graphite and mercury targets are located in series to produce pions/muons and neutrons, respectively. The DeeMe experiment uses the graphite target to produce muons, and an aluminum target that will be installed just behind the graphite target. After the aluminum target is installed, the sensitivity of the experiment is expected to reach $10^{-14}$. The DeeMe experiment plans to use a new beamline that is to be constructed in the MLF as an electron spectrometer to select only highmomentum electrons with delayed timing after the proton beam hits the graphite target. The electron energy will be precisely measured by a detector system located at the end of the beamline. A magnetic kicker system is planned to be installed in the beamline to prevent the prompt background particles from entering the electron detector.

\section{Summary}

The muon has played an important role in establishing the SM of elementary particle physics, as seen from its history. In addition, the muon is currently attracting further attention as a clue to a new physics beyond the SM. Owing to recent developments of high-power proton accelerators at advanced facilities in the world, even higher intensity muon beam is getting realistic with satisfying ultimate requirements from experiments. It is expected that muon-based cLFV search experiments performed at these new facilities will reveal the new physics beyond the SM in the near future.

\section{References}

[1] S. H. Neddermeyer and C. D. Anderson, Note on the Nature of Cosmic-Ray Particles, Physical Review 51 (1937) 884

[2] E. P. Hincks and B. Pontecorvo, Search for Gamma-Radiation in the 2.2-Microsecond Meson Decay Process, Physical Review 73 (1948) 257.

[3] G. Feinberg, Decays of the $\mu$ Meson in the Intermediate-Meson Theory, Physical Review 110 (1958) 1482.

[4] G. Danby et al., Observation of High-Energy Neutrino Reactions and the Existence of Two Kinds of Neutrinos, Physical Review Letters, 9 (1962) 36.

[5] R. Barbieri and L. J. Hall, Signals for Supersymmetric Unification, Physics Letters, B338 (1994) 212.

[6] R. Barbieri et al., Violations of Lepton Flavor and CP in Supersymmetric Unified Theories, Nuclear Physics, B 445 (1995) 219. 
[7] J. Hisano and D. Nomura, Solar and Atmospheric Neutrino Oscillations and Lepton Flavor Violation in Supersymmetric Models with Right-handed Neutrinos, Physical Review, D59 (1999) 116005 .

[8] Y. Uchiyama, Search for Lepton Flavor Violating Muon Decay: Latest Result from MEG, Presentation in this conference.

[9] The Mu2e Collaboration, Proposal to Search for $\mu^{-} N \rightarrow e^{-} N$ with a Single Event Sensitivity below $10^{-16}$, Fermilab Proposal (2008).

[10] The COMET Collaboration, An Experimental Search for Lepton Flavor Violating $\mu^{-}-e^{-}$Conversion at Sensitivity of $10^{-16}$ with a Slow-Extracted Bunched Beam, J-PARC proposal (2008).

[11] W. Burtl et al. (The SINDRUM II Collaboration), A Search for $\mu$-e Conversion in Muonic Gold, European Physical Journal, C47 (2006) 337.

[12] The DeeMe Collaboration, Proposal of an Experiment Searching for m-e Conversion in Nuclear Field at J-PARC Muon Facility, J-PARC proposal (2010). 